\title{
Mechanism of chronic dietary iron overload-induced liver damage in mice
}

\author{
DAN LIU ${ }^{1,2^{*}}$, HUAN HE$^{2 *}$, DONG YIN ${ }^{3 *}$, AILING QUE ${ }^{2}$, LEI TANG ${ }^{2}$, ZHANGPING LIAO $^{2}$, \\ QIREN HUANG ${ }^{1,2}$ and MING HE ${ }^{1,2}$ \\ ${ }^{1}$ State Key Laboratory of Food Science and Technology, Nanchang University, Nanchang 330047; \\ ${ }^{2}$ Department of Pharmacology and Molecular Therapeutics, Nanchang University School of Pharmaceutical Science; \\ ${ }^{3}$ Jiangxi Provincial Key Laboratory of Molecular Medicine, Second Affiliated Hospital of \\ Nanchang University, Nanchang 330006, P.R. China
}

Received November 1, 2012; Accepted January 28, 2013

DOI: $10.3892 / \mathrm{mmr} .2013 .1316$

\begin{abstract}
Chronic iron overload may result in hepatic fibrosis and even neoplastic transformation due to a burst of reactive oxygen species (ROS). Mitochondria have been proposed to be important in the production of ROS. The purpose of this study was to investigate the role of the mitochondrial permeability transition pore (mPTP) in the burst of ROS, and to clarify the mechanism whereby ROS induced by iron overload results in hepatic damage. It has been demonstrated that when ferrocene-induced iron-overloaded mice were fed the cyclosporin A (CsA), a specific inhibitor of the mPTP, diet $(10 \mathrm{mg}$ / $\mathrm{kg}$ /day) for 50 days, liver-to-body weight ratio, serum levels of alanine transaminase (ALT) and aspartate transaminase (AST), ROS production, mitochondrial swelling, loss of mitochondrial membrane potential $(\Delta \psi)$ and hepatocyte apoptosis decreased. However, the total antioxidant status, including superoxide dismutase (SOD), glutathione peroxidase (GSH-Px) and catalase activities, increased. The protective effect of CsA on the liver of iron-overloaded mice may be due to inhibition of the ROS burst and a successive antioxidant effect. To the best of our knowledge, these data provide the first support for the theory that ROS-induced ROS release (RIRR) may be involved in the burst of ROS in the liver and greatly contribute to the hepatic damage initiated by iron overload.
\end{abstract}

\section{Introduction}

The liver is one of the most iron-rich organs of the body. Approximately $20-30 \%$ of the body's iron is stored in

Correspondence to: Professor Ming He, Department of Pharmacology and Molecular Therapeutics, Nanchang University School of Pharmaceutical Science, Nanchang 330006, P.R. China E-mail: jxhm56@hotmail.com

*Contributed equally

Key words: iron overload, liver, mitochondrial permeability transition pore, oxidative stress, reactive oxygen species hepatocytes and reticuloendothelial macrophages, thus excessive iron accumulation is clearly observed in the liver. Numerous studies have indicated that dietary iron overload enhances hepatic fibrosis and even induces neoplastic transformation $(1,2)$. These phenomena may be associated with the role of iron in triggering oxidative stress (3). However, the molecular mechanism of iron-induced hepatic damage is poorly understood.

Mitochondria are a potential target of iron-mediated injury, due to the fact that they are intrinsically rich in iron (4). Numerous data have confirmed that mitochondrial dysfunction increases reactive oxygen species (ROS) production, with serious consequences, not only for respiratory function, but also for mitochondrial DNA transcription $(5,6)$. The role of ROS in determining cell fate during exposure to excessive iron is already known; however, the mechanism whereby the burst of ROS is induced by iron overload is not yet understood. The mitochondrial production of ROS may be involved in signal transduction pathways where an initial oxidative stress signal originating at various cell sites is amplified by the mitochondria, the phenomenon of which is termed ROS-induced ROS release (RIRR).

RIRR is generated by circuits requiring mitochondrial membrane channels, including the mitochondrial permeability transition pore (mPTP) (7). mPTPs are multi-protein complexes that are capable of forming large, non-selective pores in the inner mitochondrial membrane (8). They are directly stimulated by environmental factors, such as ROS and injury (9). When the mPTP is continuously open, it causes mitochondrial swelling, which may result in rupture of the outer mitochondrial membrane (10). Generated ROS may subsequently be released into the cytosol and trigger RIRR in the neighboring mitochondria. Thus, mitochondrion-to-mitochondrion RIRR constitutes a positive feedback mechanism for enhanced ROS production that potentially causes hepatic damage.

Due to the fact that iron is a catalyst in the Haber-Weiss reaction and is involved in the initiation of oxygen radical formation (11), we hypothesized that the toxicity of iron overload is associated with mPTP-mediated RIRR. To test this hypothesis, cyclosporin A (CsA) was used to inhibit mPTP 
opening, in order to explore the underlying mechanisms of hepatic damage induced by iron overload.

\section{Materials and methods}

Materials. Ferrocene and CsA were obtained from Sigma (St. Louis, MO, USA). AIN-93G was purchased from Dyets, Inc. (Bethlehem, PA, USA), and 2',7'-dichlorfluoresceindiacetate (DCFH-DA) and rhodomine 123 were purchased from Molecular Probes (Montluçon, France). William's medium E (WME) and fetal calf serum (FCS) were obtained from Gibco-BRL (Paisley, Scotland), while collagenase D was purchased from Boehringer Mannheim (Mannheim, Germany). The remaining chemicals were purchased from the local market. Kunming mice were purchased from Tongji Medical School, Huazhong University of Science and Technology (Wuhan, China). The animals were cared for in accordance with the Guide for the Care and Use of Laboratory Animals. The use of animals was reviewed and approved by the Nanchang University Medical College Animal Care Review Committee.

Animal model for dietary iron overload. Thirty-six male Kunming mice, initially weighing $14.7 \pm 0.7 \mathrm{~g}$, were used in the present study. Mice were randomly divided into 3 groups designated as control, iron-overloaded and CsA + iron-overloaded. The animal model for dietary iron overload in this study was similar to that described previously (12). Briefly, the iron-overloaded mice were fed for 4 months on a pellet diet (AIN-93G) supplemented with iron in the form of ferrocene, while the CsA + iron-overload group was fed the AIN-93G diet supplemented, not only with iron, but also with CsA $(10 \mathrm{mg} / \mathrm{kg})$. Control mice were fed the AIN-93G diet without iron and CsA. Possible differences in dietary consumption among the 3 groups were controlled for; the control animals received an amount of food that was equal to that which the respective treated animals consumed each day. The proportion of iron in the diet was maintained at $0.2 \%(\mathrm{w} / \mathrm{w})$ for 90 days and then decreased to $0.4 \%(\mathrm{w} / \mathrm{w})$ for the remaining 30 days. All the groups were kept at $23 \pm 2^{\circ} \mathrm{C}$ under a 12 -h dark/light cycle. Animal care in this study conformed to the National Institutes of Health (NIH) Guide for Care and Use of Laboratory Animals (NIH publication 86-23, revised 1986). Following chronic feeding, mice were euthanized by cervical dislocation, and blood was collected by cardiac puncture. The liver was immediately excised, weighed and divided for analysis as described below.

Determination of serum and liver iron concentrations. Serum iron concentration was determined using the assay based on the generation of an iron-ferrozine complex, as described previously by Galleano and Puntarulo (13). Iron concentration in the digested liver sample was measured spectrophotometrically at $535 \mathrm{~nm}$, following reaction with $2 \mathrm{mM}$ bathophenanthroline disulfonic acid (14).

Determination of serum aspartate transaminase (AST) and alanine transaminase (ALT) levels. Serum AST and ALT levels were measured using an autoanalyser (Cobas Integra 400; Holliston, MA, USA) and an ALT/AST reagent kit from Roche Diagnostics (Indianapolis, IN, USA).
Preparation of the mitochondrial fraction. Mitochondria were isolated by conventional differential centrifugation from the liver of mice that had been starved overnight. The livers were homogenized in $250 \mathrm{mM}$ sucrose, $1 \mathrm{mM}$ EGTA and $10 \mathrm{mM}$ HEPES buffer ( $\mathrm{pH}$ 7.2). The mitochondrial suspension was washed twice in the same medium containing $0.1 \mathrm{mM}$ EGTA, and the final pellet was resuspended in $250 \mathrm{mM}$ sucrose to a final protein concentration of $80-100 \mathrm{mg} / \mathrm{ml}$, measured using the Biuret method, with bovine serum albumin (BSA) as the protein standard.

Mitochondrial swelling. The swelling experiments were conducted according to the procedure performed by Beavis et al (15), at $25^{\circ} \mathrm{C}$ in a standard medium containing $125 \mathrm{mM}$ sucrose, $10 \mathrm{mM}$ HEPES buffer (pH 7.2), $2.5 \mathrm{mM}$ succinate and $4.0 \mathrm{mM}$ rotenone. The final volume used was $1.0 \mathrm{ml}$, and the protein concentration was $\sim 0.5 \mathrm{mg} / \mathrm{ml}$. Absorbance changes at $520 \mathrm{~nm}$ were monitored in a thermostatically controlled Hitachi U-2000 spectrophotometer.

Hepatocyte preparation. Hepatocytes were isolated by a two-step collagenase perfusion method. Following mechanical disruption of the liver capsule, the liver cells were collected in WME and serially filtered through 30-, 50- and 80-mesh filters in an 85-ml Cellector tissue sieve (Bellco Biotechnology, Vineland, NJ, USA). Typically, $10-25 \times 10^{6}$ cells were obtained from one mouse liver.

Measurements of intracellular $\Delta \psi$. The mitochondrial membrane potential $(\Delta \psi)$ was measured by flow cytometry using rhodomine 123 , a fluorescent dye that has been demonstrated to selectively accumulate in the mitochondria of living hepatocytes by a mechanism that is dependent on the $\Delta \psi(16)$. The hepatocytes were resuspended in $0.5 \mathrm{ml}$ of $10 \mu \mathrm{g} /$ $\mathrm{ml}$ rhodomine for $15 \mathrm{~min}$ at $37^{\circ} \mathrm{C}$, and were immediately submitted for flow analysis.

Measurement of intracellular ROS production. To assess the intracellular ROS levels, flow cytometric analyses were performed using the oxidative-sensitive fluorescent probe, DCFH-DA, as previously described (17). Hepatocytes were incubated with $10 \mu \mathrm{M} \mathrm{DCFH}-\mathrm{DA}$ for $30 \mathrm{~min}$ at $37^{\circ} \mathrm{C}$. Formation of $2^{\prime}, 7^{\prime}$-dichlorofluorescein (DCF) was then detected using a FACSCalibur (Becton-Dickinson, Mountain View, CA, USA) equipped with an argon laser $(488 \mathrm{~nm})$ in the FL1 channel.

\section{Determination of oxidative stress parameters and lipid peroxidation}

$M D A$. The level of 3,4-methylenedioxyamphetamine (MDA) was determined according to the procedure employed by Okhawa et al (18). Briefly, $0.5 \mathrm{ml}$ supernatant was mixed with $1.5 \mathrm{ml}$ thiobarbituric acid, $1.5 \mathrm{ml}$ acetic acid $(\mathrm{pH} 3.5), 0.2 \mathrm{ml}$ sodium dodecyl sulfate and $0.5 \mathrm{ml}$ distilled water. Following mixing, the samples and standards were heated at $100^{\circ} \mathrm{C}$ for $1 \mathrm{~h}$. The absorbance was recorded at $532 \mathrm{~nm}$ and compared with that of the MDA standards.

$S O D$. Superoxide dismutase (SOD) activity was determined according to the method utilised by Beauchamp and Fridovich (19). The reaction mixture consisted of $100 \mu \mathrm{mol} / 1$ xanthine, $100 \mu \mathrm{mol} / \mathrm{l}$ EDTA, $25 \mu \mathrm{mol} / \mathrm{l} \mathrm{NBT}$ and $50 \mathrm{mmol} / \mathrm{l}$ 
$\mathrm{Na}_{2} \mathrm{CO}_{3}$ ( $\mathrm{pH}$ 10.2). The reaction was initiated by the addition of xanthine oxidase, and then the absorbance at $560 \mathrm{~nm}$ was read every $30 \mathrm{sec}$ for $5 \mathrm{~min}$. SOD activity was assayed spectrophotometrically as the inhibition of the photochemical reduction of NBT at $560 \mathrm{~nm}$.

GSH-Px. Glutathione peroxidase (GSH-Px) activity was measured according to the method employed by Lawrence and Burk (20). The assay reaction comprised $50 \mathrm{mmol} / 1 \mathrm{~K}_{2} \mathrm{HPO}_{4}$ buffer, $1 \mathrm{mmol} / 1$ EDTA, $1 \mathrm{mmol} / 1 \mathrm{NaN}_{3}, 1 \mathrm{mmol} / 1$ reduced glutathione, $0.2 \mathrm{mmol} / 1 \mathrm{NADPH}, 0.25 \mathrm{mmol} / 1 \mathrm{H}_{2} \mathrm{O}_{2}$ and $1 \mathrm{U} /$ $\mathrm{ml}$ glutathione reductase. GSH-Px activity was assayed by monitoring NADPH oxidation at $340 \mathrm{~nm}$, by measuring the absorbance every $15 \mathrm{sec}$ for $5 \mathrm{~min}$. The activity was calculated using a molar extinction coefficient for NADPH of $6.22 \mathrm{mM}^{-1} \mathrm{~cm}^{-1}$ at $340 \mathrm{~nm}$.

Catalase. Catalase activity in the liver homogenate was assayed using a modification of the procedure described by Pedraza-Chaverri et al (21). The catalase activity of hepatic homogenates was assayed at $25^{\circ} \mathrm{C}$, based on the disappearance of $10 \mathrm{mM} \mathrm{H}_{2} \mathrm{O}_{2}$ at $240 \mathrm{~nm}$. The results are expressed as U/mg protein.

TUNEL assay. The terminal deoxynucleotidyl transferase-mediated nick-end labeling (TUNEL) assay was performed to detect hepatocyte apoptosis. The hepatocytes were plated on glass Lab-Tek Chamber slides (Sigma) and washed with PBS, then fixed in $1 \%$ paraformaldehyde for 10 $\min$. These were then postfixed in pre-cooled ethanol-acetic acid $(2: 1)$ for a further $5 \mathrm{~min}$ at $-20^{\circ} \mathrm{C}$. Following washing with PBS, the cells were incubated with a TUNEL reaction buffer at $37^{\circ} \mathrm{C}$ for $1 \mathrm{~h}$ in a humidified chamber. As a positive control, cells were treated with DNase I (1.0 mg/ml, Sigma) for $10 \mathrm{~min}$, to introduce nicks into the genomic DNA. The percentage of cardiomyocytes with DNA nick-end labeling was determined by counting the number of cells exhibiting brown nuclei among 1,000 cells in duplicate plates.

Statistical analysis. Values were expressed as the mean \pm standard deviation from $\geq 12$ independent experiments. Each treatment was performed in triplicate culture wells. The differences in the means between each group were tested by one-way ANOVA followed by the Student-Newman-Keuls test (comparison between multiple groups). $\mathrm{P}<0.05$ was considered to indicate a statistically significant difference.

\section{Results}

Effects of CsA administration on serum and hepatic iron concentrations in iron-overloaded mice. As expected, the serum and hepatic iron concentrations were significantly increased in all treated animals. When mice were supplemented with CsA, the serum and hepatic iron concentrations were not significantly different compared with those of the iron-overloaded group. This observation suggests that the severe iron loading caused by a continuous iron-supplemented diet was not alleviated by CsA aministration (Fig. 1).

Effects of CsA administration on liver-to-body weight ratio (\%) and serum levels of transaminases in iron-overloaded mice. There were no obvious health abnormalities
A

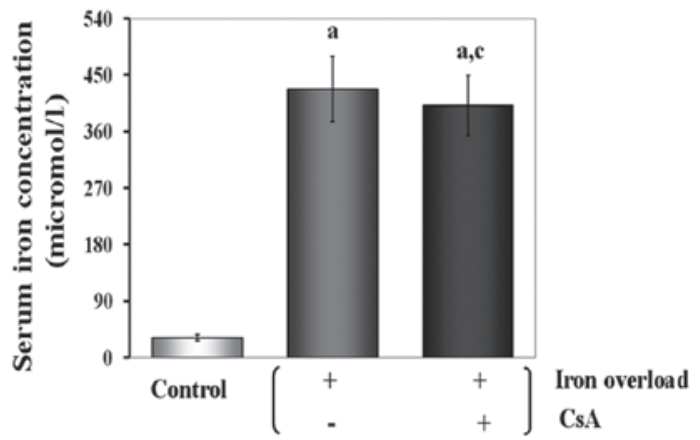

B

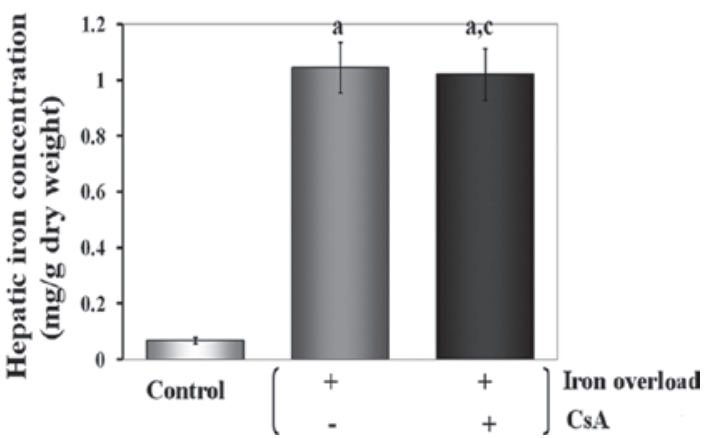

Figure 1. Effects of cyclosporin A (CsA) on serum and hepatic iron concentrations in iron-overloaded mice. Mice were randomly divided into 3 groups and treated as described in Materials and methods. (A) Non-heme iron content in the serum of mice in the different treatment groups. The blood was collected by cardiac puncture. Serum iron concentration was determined using the assay based on the generation of an iron-ferrozine complex. (B) Liver iron content in the liver of mice in the different treatment groups. The liver was immediately removed and weighed, then digested by nitric acid and perchloric acid mixed at a ratio of 4:1. Iron content was assayed. Values are the mean \pm standard deviation ( $\mathrm{n}=12$ ). ${ }^{\mathrm{a}} \mathrm{P}<0.01$ vs. control; ${ }^{\mathrm{C}} \mathrm{P}>0.05$ vs. iron-overloaded group.

in any of the groups, but the liver-to-body weight ratio was significantly increased in all the treated animals. Compared with the iron-overloaded group, Cs A showed significant protection against iron overload in the CsA + iron-overloaded group $(\mathrm{P}<0.01)$.

Serum levels of transaminases (ALT and AST) were used as indicators to evaluate the involvement of CsA in the structural damage to the liver. In this experiment, the enzyme assays of serum transaminases demonstrated that the iron overload significantly raised the levels of ALT and AST to 227.1 and $381.3 \mathrm{U} / 1$, respectively ( $\mathrm{P}<0.01$ for both). CsA was capable of effectively inhibiting the enzyme activity. The levels of ALT and AST were reduced to 125.5 and $220.1 \mathrm{U} / 1$, respectively ( $\mathrm{P}<0.01$ for both) when CsA was administered. This result revealed that the negative effect of iron overload in mice may be alleviated by CsA administration (Fig. 2).

Effects of CsA administration on hepatocyte apoptosis in iron-overloaded mice. In the control group, a limited number of TUNEL-positive hepatocytes were detected. By contrast, numerous hepatocytes in the iron-overloaded group presented as positive for TUNEL, and this number was significantly greater than that of the control group $(\mathrm{P}<0.01)$. The number of TUNEL-positive cells was significantly reduced $(10.4 \pm 2.1 \%)$ compared with the iron-overloaded group when CsA was administered $(\mathrm{P}<0.01)$ (Fig. 3). 
Table I. Effects of CsA on oxidative stress parameters in iron-overloaded mice.

\begin{tabular}{llcc}
\hline Index & Control & Iron-overloaded & CsA + iron-overloaded \\
\hline SOD activity (U/mg protein) & $382.2 \pm 15.5$ & $120.1 \pm 5.0^{\mathrm{a}}$ & $302.6 \pm 13.0^{\mathrm{b}}$ \\
GSH-Px activity (mU/mg protein) & $248.4 \pm 8.9 .$. & $108.6 \pm 4.0^{\mathrm{a}}$ & $160.3 \pm 6.2^{\mathrm{a}, \mathrm{b}}$ \\
Catalase activity (U/mg protein) & $316.2 \pm 14.5$ & $186.3 \pm 6.5^{\mathrm{a}}$ & $271.6 \pm 8.7^{\mathrm{b}} .$. \\
MDA content (pmol/mg protein) & $130.4 \pm 4.4 .$. & $518.5 \pm 21.1^{\mathrm{a}}$ & $281.2 \pm 10.3^{\mathrm{a}, \mathrm{b}}$
\end{tabular}

CsA, cyclosporin A; SOD, superoxide dismutase; GSH-Px, glutathione peroxidase; MDA, 3,4-methylenedioxyamphetamine. Data are expressed as the mean \pm standard deviation $(n=12)$. ${ }^{a} \mathrm{P}<0.01$ vs. control; ${ }^{b} \mathrm{P}<0.01 \mathrm{vs}$. iron-overloaded group.

$\mathbf{A}$

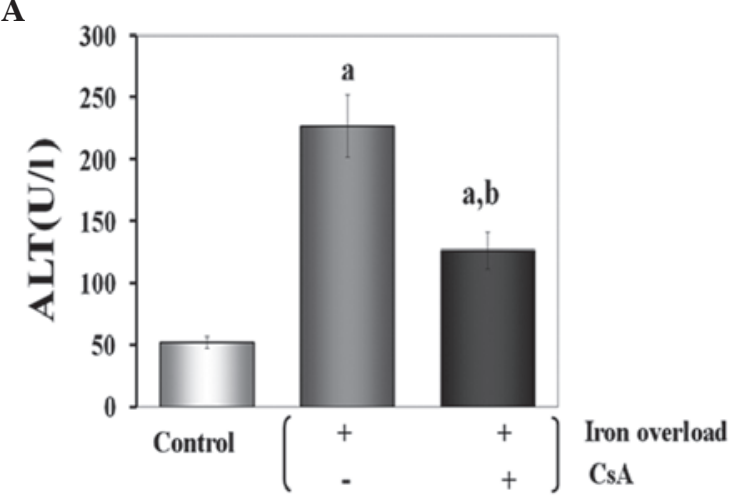

B

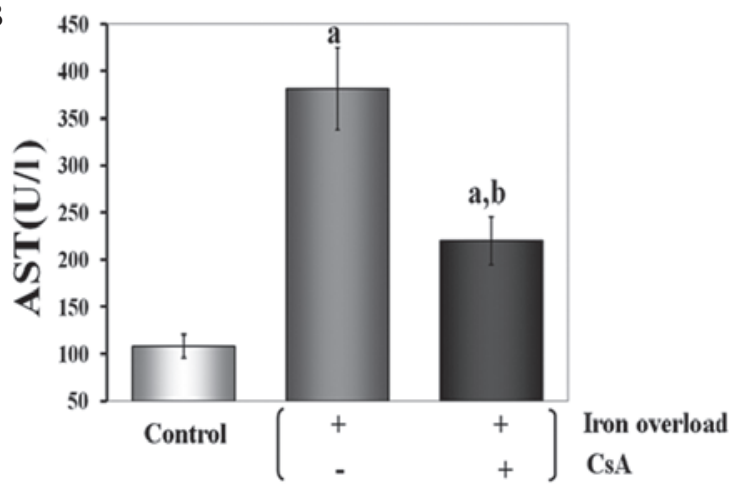

c

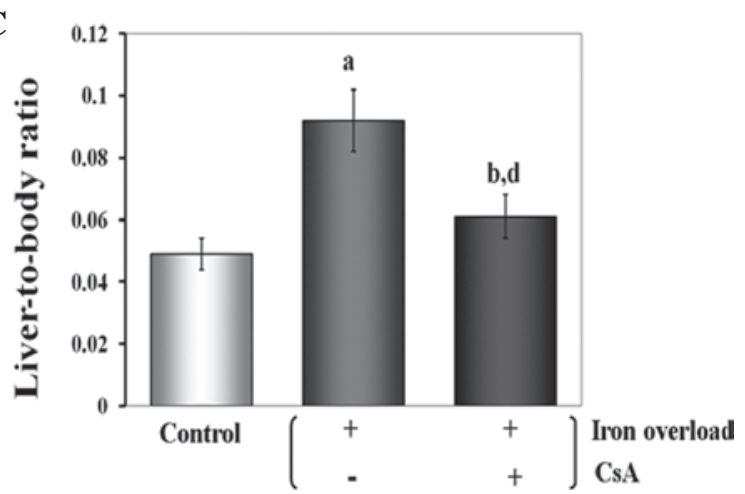

Figure 2. Effects of cyclosporin A (CsA) on the liver-to-body weight ratio (\%) and serum levels of transaminases of iron-overloaded mice. Mice were randomly divided into 3 groups and treated as described in Materials and methods. (A and B) Alanine transaminase (ALT) and aspartate transaminase (AST) activities in the serum of mice in the different treatment groups. The blood was collected by cardiac puncture. Serum AST and ALT levels were measured as described in Materials and methods. (C) Liver-to-body weight ratio of mice in the different treatment groups. The liver was immediately removed and weighed and the liver-to-body weight ratio was calculated. Values are the mean \pm standard deviation $(\mathrm{n}=12) .{ }^{\mathrm{a}} \mathrm{P}<0.01$ vs. control; ${ }^{\mathrm{b}} \mathrm{P}<0.01$ vs. iron-overloaded group; ${ }^{\mathrm{d}} \mathrm{P}<0.05$ vs. control and iron-overloaded groups.
Effects of CsA administration on the oxidative parameters of iron-overloaded mice. The effects of CsA on the oxidative stress of iron-overloaded mice $(n=12)$ were estimated by determining the activities of MDA, SOD, GSH-Px and catalase in the liver tissue. The MDA level is a key marker of endogenous lipid peroxidation. In the iron-overloaded group, the MDA level increased significantly in the liver compared with the control group $(\mathrm{P}<0.01)$. By contrast, the MDA level in the CsA-treated group decreased significantly compared with the ferrocene-treated group $(\mathrm{P}<0.01)$. This revealed that CsA was able to successfully block lipid peroxidation. SOD, GSH-Px and catalase are intracellular antioxidant enzymes that protect against oxidative processes. As shown in Table I, iron overload induced severe oxidative damage and the SOD, GSH-Px and catalase levels decreased markedly, while CsA effectively normalized the enzyme activities.

Effects of CsA administration on mitochondrial swelling in iron-overloaded mice. Iron-induced damage to the inner mitochondrial membrane may be assessed by the classic swelling techniques, which monitor the net influx of the osmotic support that is associated with a non-specific increase in membrane permeability. It was demonstrated that iron-dextran induced mitochondrial swelling, as revealed by the large decrease in the absorbance of the mitochondrial suspension at $520 \mathrm{~nm}$. However, CsA inhibited the swelling process $(\mathrm{P}<0.01)$ (Fig. 4).

Effects of CsA administration on $\Delta \psi$ of iron-overloaded mice. The $\Delta \psi$ was determined by the $\Delta \psi$-sensitive fluorescent probe, rhodimine 123. The findings revealed that iron overload induced $\Delta \psi$ depolarization and CsA prevented $\Delta \psi$ dissipation (Fig. 5).

Effects of CSA administration on ROS production in iron-overloaded mice. By flow cytometry, using the DCFH-DA fluorescent probe, it was demonstrated that iron-dextran induced ROS production. As expected, iron overload induced a ROS burst, while ROS overproduction was prevented when CsA was added simultaneously to iron $(\mathrm{P}<0.01)$ (Fig. 6).

\section{Discussion}

Iron is an essential micronutrient. The capacity of readily exchanging electrons under aerobic conditions causes iron to be essential for fundamental cell functions, such as DNA 

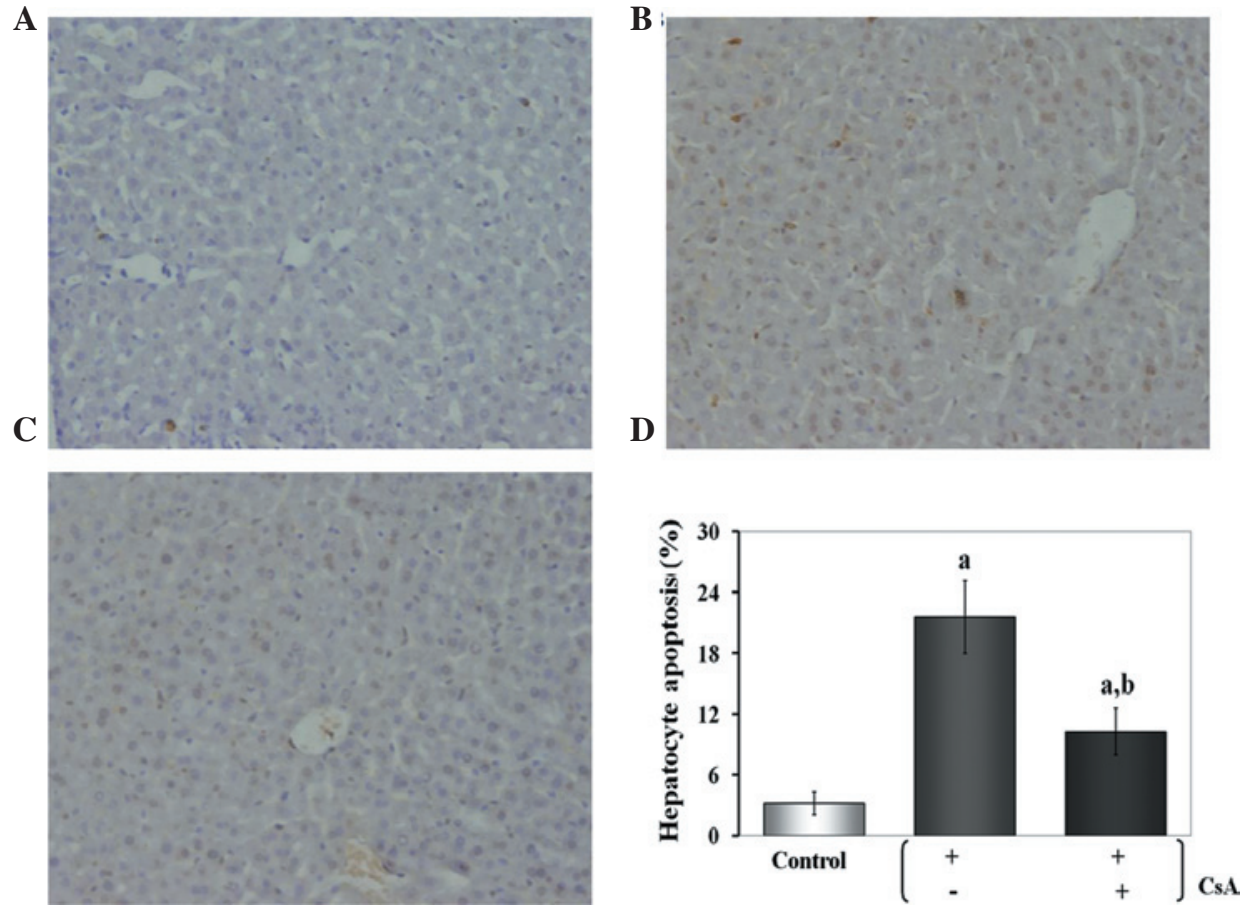

Figure 3. Effects of cyclosporin A (CsA) on liver apoptosis in iron-overloaded mice. Following 4 months of feeding, mice were euthanized by cervical dislocation. Livers were immediately removed and fixed by perfusion with $10 \%$ buffered formalin, and $5-\mu \mathrm{m}$ paraffin-embedded sections were obtained Subsequently, the sections were subjected to terminal deoxynucleotidyl transferase-mediated nick-end labeling (TUNEL) assay. (A-C) Detection of apoptotic hepatocytes by TUNEL in the different groups: (A) control, (B) iron-overloaded and (C) CsA + iron-overloaded. Representative photomicrographs show TUNEL-positive apoptotic hepatocytes. Original magnification, x200. (D) Quantitative analysis of apoptotic hepatocytes expressed as the percentage of TUNEL-positive nuclei in hepatocytes. Values are the mean \pm standard deviation $(\mathrm{n}=12)$. ${ }^{\mathrm{a}} \mathrm{P}<0.01 \mathrm{vs}$. control; ${ }^{\mathrm{b}} \mathrm{P}<0.01 \mathrm{vs}$. iron-overloaded group.

A

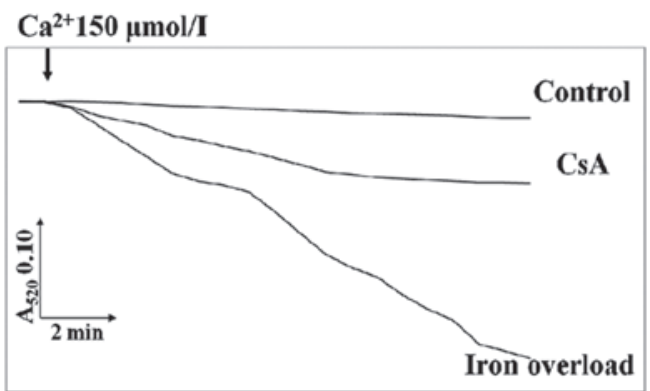

B

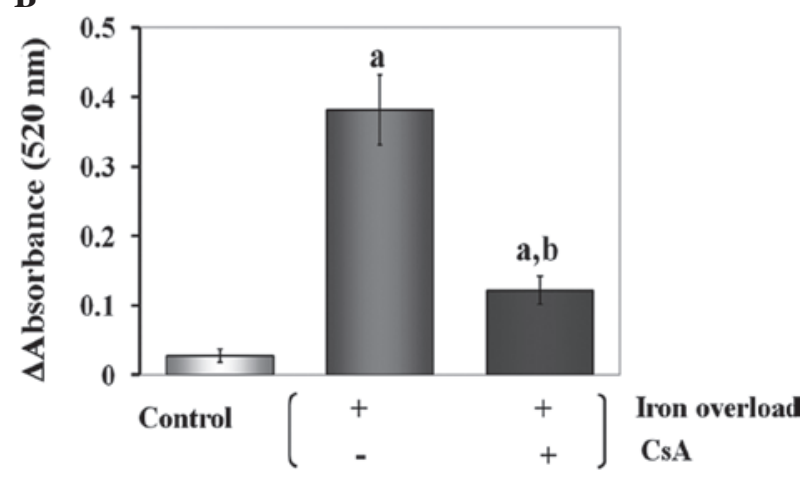

Figure 4. Effects of cyclosporin A (CsA) on the mitochondrial swelling of iron-overloaded mice. Mitochondria were isolated by conventional differential centrifugation from the liver of mice. The swelling experiments were conducted as decribed in Materials and methods. (A) Effect of CsA and iron overload on $\mathrm{Ca}^{2+}$-induced mitochondrial swelling. During the onset of mitochondrial swelling, $150 \mu \mathrm{M} \mathrm{Ca}^{2+}$ was added to initiate the reaction. (B) Quantitative analysis of mitochondrial swelling. Values are the mean \pm standard deviation $(\mathrm{n}=12) .{ }^{\mathrm{a}} \mathrm{P}<0.01$ vs. control; ${ }^{\mathrm{b}} \mathrm{P}<0.01 \mathrm{vs}$. iron-overloaded group. synthesis, transport of oxygen and electrons, and cell respiration (22). However, as humans have no means to control their iron excretion, excess iron consumed in the diet accumulates in parenchymal organs and threatens cell viability (23). In the current study, in mice fed a diet supplemented with ferrocene, severe iron overload occurred and cell damage arose mainly in the liver (the body's main storage site for iron). In the present study, a specific inhibitor of mPTP, CsA, was used (24). Notably, CsA was unable to reduce iron accumulation in the liver, but it was able to protect the liver from iron overload.

The MPTP is a voltage-dependent, high-conductance channel located in the inner mitochondrial membrane, which has been suggested to be formed by the interaction of several proteins that connect the mitochondrial matrix to the cytosolic space (25). It has been proposed that the MPTP is able to open in two modes: low and high conductance (26). When mPTPs are opened in the long-lasting high-conductance mode, necrosis and apoptosis are initiated (27). In the physiological setting, ROS is the most important inducer of mPTP opening (28).

The chemical structure of iron and its ability to drive one-electron reactions causes iron to be key for the production and metabolism of ROS in biological systems.A previous study clearly demonstrated that these pathological events are induced by iron-generated ROS (29). As demonstrated in the present study, such an iron burden initiates significant oxidative stress. We found that the production of ROS was greater in the iron-overloaded group compared with the control group. ROS 
A

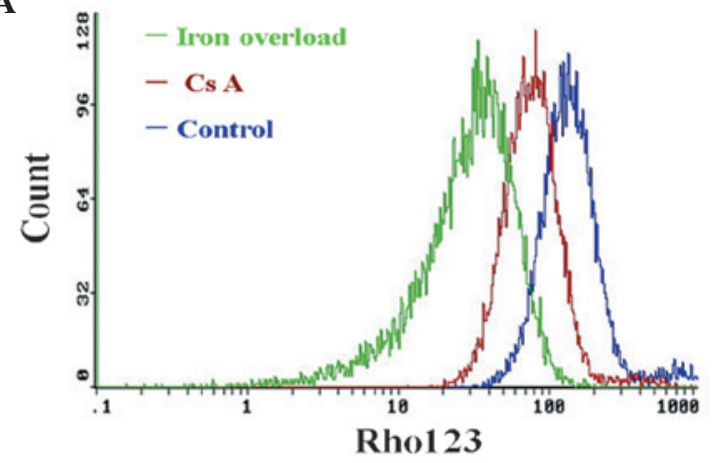

B

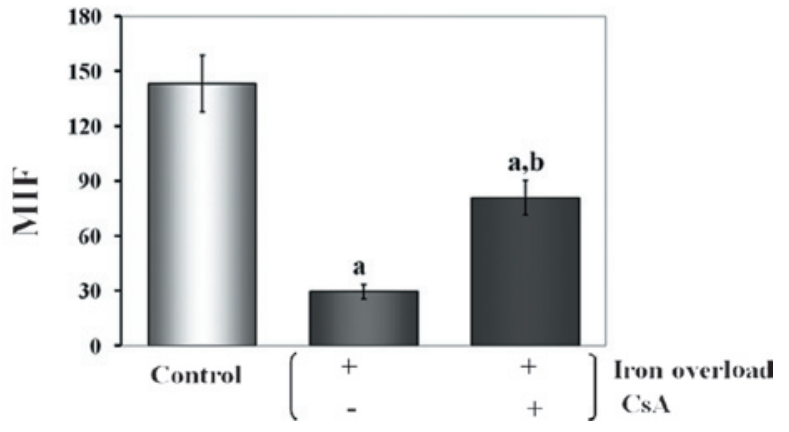

Figure 5. Effects of cyclosporin A (CsA) on the mitochondrial membrane potential $(\Delta \psi)$ of iron-overloaded mice. Livers were removed and hepatocytes were isolated by a two-step collagenase perfusion method. (A) Effects of CsA or iron overload on the $\Delta \psi$. Hepatocytes were immediately collected, resuspended in PBS, stained with Rho 123 for 15 min and subjected to flow cytometry. (B) Quantitative analysis of $\Delta \psi$. Values are the mean \pm standard deviation ( $\mathrm{n}=12)$. ${ }^{\mathrm{a}} \mathrm{P}<0.01$ vs. control; ${ }^{\mathrm{b}} \mathrm{P}<0.01$ vs. iron-overloaded group.

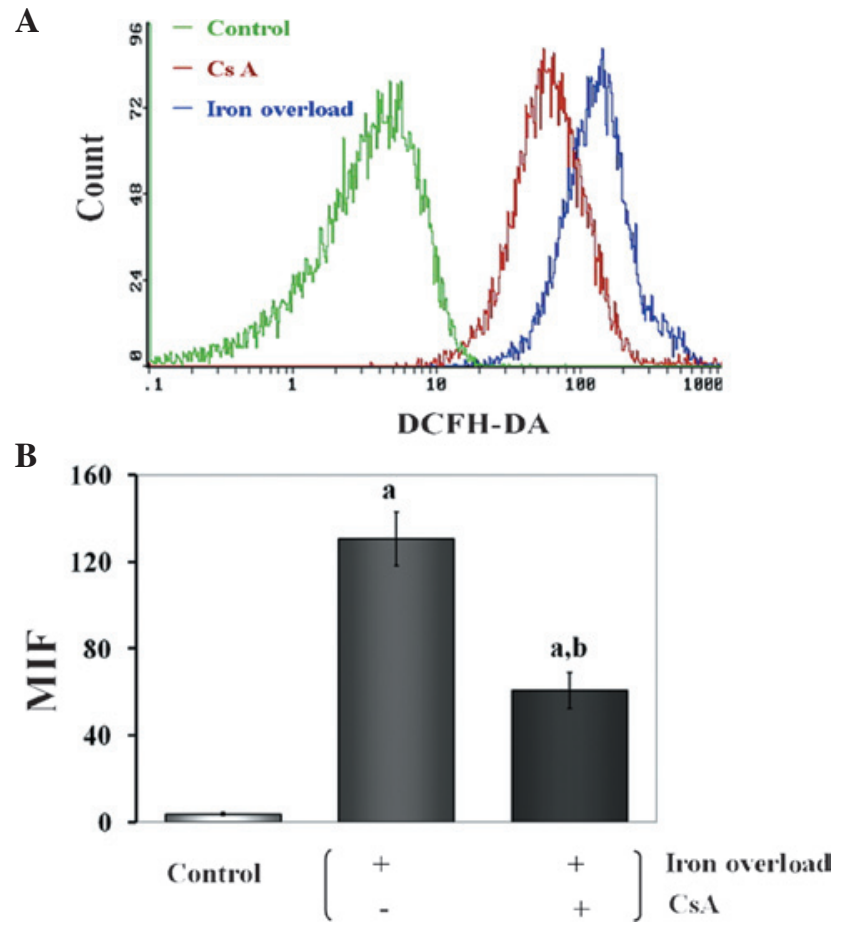

Figure 6. Effects of cyclosporin A (CsA) on reactive oxygen species (ROS) production in iron-overloaded mice. (A) Effects of CsA or iron overload on intracellular ROS levels in hepatocytes. The figure shows the overlay of three representative fluorescence flow cytometric analyses performed in hepatocytes. (B) Quantitative analysis of ROS production. Values are the mean \pm standard deviation $(\mathrm{n}=12)$. ${ }^{\mathrm{a}} \mathrm{P}<0.01 \mathrm{vs}$. control; ${ }^{\mathrm{b}} \mathrm{P}<0.01$ vs. iron-overloaded group. are capable of causing oxidative damage to macromolecules, leading to lipid peroxidation, oxidation of amino acid side chains (particularly cysteine), formation of protein-protein cross-links and oxidation of polypeptide backbones, resulting in protein fragmentation, hepatic cell apoptosis and even necrosis (30).

Iron, as a transition metal catalyst, is essential for the initiation step in the generation of ROS. Subsequently, excess ROS are the major cause of liver damage. Nevertheless, the mechanism of the burst of ROS induced by iron overload has not yet been clarified. Recent data have confirmed that mitochondria are also capable of producing a significant ROS release when the $\Delta \psi$ is null following mPTP opening $(31,32)$. Additionally, ROS are the most important inducer of mPTP opening (33). These transitions have been described and occur via mechanisms involved in the process of RIRR (31,32). Although the phenomenon of RIRR initiated by ischemic reperfusion injury has been confirmed in cardiomyocytes (34), RIRR initiated by iron overload has not yet been demonstrated in hepatocytes. We suggest that under conditions that lead to RIRR, such as exposure to iron overload, the increase in ROS reaches a threshold level triggering mPTP opening, which in turn leads to the simultaneous collapse of $\Delta \psi$ and a transient increase in ROS generation by the electron transfer chain (35). The release of this ROS burst into the cytosol may potentially function as a second messenger to activate RIRR in the neighboring mitochondria. Thus, mitochondrion-to-mitochondrion RIRR constitutes a positive feedback mechanism for enhanced ROS production, potentially leading to significant mitochondrial and cell injury. RIRR is generated by circuits requiring mitochondrial membrane channels, including the mPTP. Therefore, the mPTP is important in RIRR. Our present results have demonstrated that the large-amplitude ROS burst initiated by iron overload was prevented by the administration of CsA. Moreover, depolarization and apoptosis were prevented, while inhibition of the ROS burst in mice was estimated by determining the activities of MDA, SOD, GSH-Px, catalase, ALT and AST in serum and tissues. CsA not only protected the liver from damage by efficiently inhibiting MDA formation, and by reducing AST and ALT, but it also enhanced the activities of the antioxidant enzyme system of the host, including those of SOD, GSH-Px and catalase.

Similar to the propagation of mitochondrial depolarization and ROS production demonstrated in isolated cardiomyocytes by Zorov et al (34), it has been observed that mPTP-mediated RIRR also exists in liver subjected to iron overload. Iron overload initiates the generation of ROS, and ROS induce mPTP opening. Extensive matrix swelling with long-lasting mPTP opening may lead to the unfolding of cristae, causing the outer membrane to rupture, irreversibly damaging the mitochondria, and consequently, ROS are released from the mitochondrial matrix into the cytosol. Thus, ROS may potentially function as a second messenger to activate RIRR in the neighboring mitochondria, and the liver overloaded with iron becomes damaged.

In conclusion, our results strongly support the hypothesis that RIRR mediated by mPTP may generate a large number of ROS, and provide a possible mechanism by which excess dietary iron uptake results in liver damage in mice. 


\section{Acknowledgements}

This study was supported by grants from the Natural Scientific Foundations of China (grant nos. 30760075, 30860271 and 81100104).

\section{References}

1. Nahon P, Ganne-Carrié N, Trinchet JC and Beaugrand M: Hepatic iron overload and risk of hepatocellular carcinoma in cirrhosis. Gastroenterol Clin Biol 34: 1-7, 2010.

2. Allen KJ, Gurrin LC, Constantine CC, et al: Iron-overload-related disease in HFE hereditary hemochromatosis. N Engl J Med 358: 221-230, 2008

3. Asare GA, Kew MC, Mossanda KS, Paterson AC, Siziba K and Kahler-Venter CP: Effects of exogenous antioxidants on dietary iron overload. J Clin Biochem Nutr 44: 85-94, 2009.

4. Pardo Andreu GL, Inada NM, Vercesi AE and Curti C: Uncoupling and oxidative stress in liver mitochondria isolated from rats with acute iron overload. Arch Toxicol 83: 47-53, 2009.

5. Starkov AA: The role of mitochondria in reactive oxygen species metabolism and signaling. Ann NY Acad Sci 1147: $37-52,2008$

6. Murphy MP: How mitochondria produce reactive oxygen species. Biochem J 417: 1-13, 2009.

7. Brady NR, Elmore SP, van Beek JJ, Krab K, Courtoy PJ, Hue L and Westerhoff HV: Coordinated behavior of mitochondria in both space and time: a reactive oxygen species-activated wave of mitochondrial depolarization. Biophys J 87: 2022-2034, 2004.

8. Gateau-Roesch O, Argaud L and Ovize M: Mitochondrial permeability transition pore and postconditioning. Cardiovasc Res 70: 264-273, 2006

9. Saotome M, Katoh H, Yaguchi Y, Tanaka T, Urushida T, Satoh H and Hayashi H: Transient opening of mitochondrial permeability transition pore by reactive oxygen species protects myocardium from ischemia-reperfusion injury. Am J Physiol Heart Circ Physiol 296: H1125-H1132, 2009.

10. Halestrap AP: Calcium, mitochondria and reperfusion injury: a pore way to die. Biochem Soc Trans 34: 232-237, 2006.

11. Galaris D, Skiada V and Barbouti A: Redox signaling and cancer: the role of 'labile' iron. Cancer Lett 266: 21-29, 2008

12. Tjalkens RB, Valerio LG Jr, Awasthi YC and Petersen DR: Association of glutathione S-transferase isozyme-specific induction and lipid peroxidation in two inbred strains of mice subjected to chronic dietary iron overload. Toxicol Appl Pharmacol 151: 174-181, 1998.

13. Galleano M and Puntarulo S: Hepatic chemiluminescence and lipid peroxidation in mild iron overload. Toxicology 76: 27-38, 1992.

14. Brumby PE and Massey V: Determination of nonheme iron, total iron, and copper. Methods Enzymol 10: 463-474, 1967.

15. Beavis AD, Brannan RD and Garlid KD: Swelling and contraction of the mitochondrial matrix. I. A structural interpretation of the relationship between light scattering and matrix volume. J Biol Chem 260: 13424-13433, 1985.

16. Emaus RK, Grunwald R and Lemasters JJ: Rhodamine 123 as a probe of transmembrane potential in isolated rat-liver mitochondria: spectral and metabolic properties. Biochim Biophys Acta 850: 436-448, 1986.

17. Desmots F, Rissel M, Pigeon C, Loyer P, Loréal O and Guillouzo A: Differential effects of iron overload on GST isoform expression in mouse liver and kidney and correlation between GSTA4 induction and overproduction of free radicals. Free Radic Biol Med 32: 93-101, 2002.
18. Okhawa $\mathrm{H}$, Ohishi $\mathrm{N}$ and Yagi K: Assay for lipid peroxides in animal tissues by thiobarbituric acid reaction. Anal Biochem 95: 351-358, 1979.

19. Beauchamp C and Fridovich I: Superoxide dismutase: improved assays and an assay applicable to acrylamide gels. Anal Biochem 44: 276-287, 1971.

20. Lawrence RA and Burk RF: Glutathione peroxidase activity in selenium-deficient rat liver. Biochem Biophys Res Commun 71: 952-958, 1976.

21. Pedraza-Chaverri J,Granados-Silvestre MD,Medina-Campos ON and Hernández-Pando R: Effect of the in vivo catalase inhibition on aminonucleoside nephrosis. Free Radic Biol Med 27: 245-253, 1999.

22. Ramm GA and Ruddell RG: Iron homeostasis, hepatocellular injury, and fibrogenesis in hemochromatosis: the role of inflammation in a noninflammatory liver disease. Semin Liver Dis 30: 271-287, 2010.

23. Chen J and Chloupková M: Abnormal iron uptake and liver cancer. Cancer Biol Ther 8: 1699-1708, 2009.

24. Xie JR and Yu LN: Cardioprotective effects of cyclosporine A in an in vivo model of myocardial ischemia and reperfusion. Acta Anaesthesiol Scand 51: 909-913, 2007.

25. Halestrap AP, McStay GP and Clarke SJ: The permeability transition pore complex: another view. Biochimie 84: 153-166, 2002.

26. Novgorodov SA and Gudz TI: Permeability transition pore of the inner mitochondrial membrane can operate in two open states with different selectivities. J Bioenerg Biomembr 28: 139-146, 1996.

27. Joza N, Susin SA, Daugas E, et al: Essential role of the mitochondrial apoptosis-inducing factor in programmed cell death. Nature 410: 549-554, 2001.

28. Halestrap AP: The mitochondrial permeability transition - a'pore way for the heart to die. J Clin Basic Cardiol 5: 29-41, 2002.

29. Galaris D and Pantopoulos K: Oxidative stress and iron homeostasis: mechanistic and health aspects. Crit Rev Clin Lab Sci 45: $1-23,2008$

30. Van HB, Woshner V and Santos JH: Role of mitochondrial DNA in toxic responses to oxidative stress. DNA Repair (Amst) 5: $145-152,2006$

31. Zorov DB, Juhaszova M and Sollott SJ: Mitochondrial ROS-induced ROS release: an update and review. Biochim Biophys Acta 1757: 509-517, 2006.

32. Brady NR, Hamacher-Brady A, Westerhoff HV and Gottlieb RA: A wave of reactive oxygen species (ROS)-induced ROS release in a sea of excitable mitochondria. Antioxid Redox Signal 8: 1651-1665, 2006.

33. Serviddio G, Bellanti F, Sastre J, Vendemiale G and Altomare E: Targeting mitochondria: a new promising approach for the treatment of liver diseases. Curr Med Chem 17: 2325-2337, 2010.

34. Zorov DB, Filburn CR, Klotz LO, Zweier JL and Sollott SJ: Reactive oxygen species (ROS)-induced ROS release: a new phenomenon accompanying induction of the mitochondrial permeability transition in cardiac myocytes. J Exp Med 192: $1001-1014,2000$

35. Batandier C, Leverve $\mathrm{X}$ and Fontaine E: Opening of the mitochondrial permeability transition pore induces reactive oxygen species production at the level of the respiratory chain complex I. J Biol Chem 279: 17197-17204, 2004. 